\title{
BMJ Open Interventions for migraine prophylaxis: protocol of an umbrella systematic review and network meta-analysis
}

\author{
Hui Zheng, ${ }^{1}$ Min Chen, ${ }^{2}$ Dequan Huang, ${ }^{2}$ Juan Li, ${ }^{1}$ Qin Chen, ${ }^{3}$ Jianqiao Fang ${ }^{3}$
}

To cite: Zheng $\mathrm{H}$, Chen $\mathrm{M}$, Huang D, et al. Interventions for migraine prophylaxis: protocol of an umbrella systematic review and network meta-analysis. BMJ Open 2015;5:e007594. doi:10.1136/bmjopen-2015007594

- Prepublication history for this paper is available online. To view these files please visit the journal online (http://dx.doi.org/10.1136/ bmjopen-2015-007594).

Received 6 January 2015 Accepted 23 January 2015

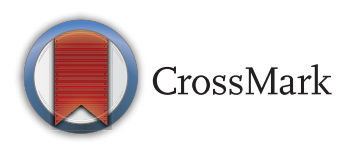

${ }^{1}$ Acupuncture \& Tuina College/The 3rd Teaching Hospital, Chengdu University of Traditional Chinese Medicine, Chengdu, China

${ }^{2}$ Anorectal Department, The First Affiliated Hospital of Chengdu University of

Traditional Chinese Medicine, Chengdu, China

${ }^{3}$ The Third Clinical College of Zhejiang Chinese Medical University, Hangzhou, China

Correspondence to Professor Jianqiao Fang; jianqiaofangzjm@126.com.

\section{ABSTRACT}

Introduction: Multiple interventions are effective for migraine prophylaxis. However, the comparative effectiveness of these interventions is still not clear. Therefore, the aim of this study is to summarise the direct and indirect evidence for pharmacological and non-pharmacological interventions to prevent migraine attack.

Methods and analysis: We will perform an umbrella systematic review to identify eligible randomised controlled trials (RCTs) for the recommended interventions for migraine prophylaxis according to the guidelines. A comprehensive literature search will be conducted in MEDLINE, EMBASE and the Cochrane library for systematic reviews, which will be screened for RCTs. We will describe the general information of the RCTs for participants, interventions, outcome measurements, comparisons and the primary findings. Additionally, a network meta-analysis will be conducted to determine the comparative effectiveness of the treatments with a random-effects model. The absolute and relative effectiveness of the treatments will be provided. The heterogeneity and inconsistency between trials will be assessed by the $\mathrm{I}^{2}$ statistical test and Cochrane's $Q$ test. Risk of bias will be assessed and the overall strength of the evidence will be summarised.

Discussion: The result of this network meta-analysis will provide direct and indirect evidence of treatments for migraine prophylaxis, and it may provide a ranking of the treatments for patients and clinicians to help them select the best option.

Trial registration number: PROSPERO CRD42015015297.

\section{INTRODUCTION}

Migraine is a primary headache with a high prevalence in the global population. About $6 \%$ of the males and $16 \%$ of the females in the USA and England reported experience of migraine attacks. ${ }^{1-3}$ Lower prevalence was found in Hong Kong, Taiwan and Mainland China, but it still reaches $4.7 \%, 9.1 \%$ and $9.3 \%$, respectively. ${ }^{4-6}$ Both episodic and chronic migraine headaches are associated with lower work productivity ${ }^{7}$ and lower

\section{Strengths and limitations of this study}

To the best of our knowledge, this is the first network meta-analysis protocol to study the comparative effectiveness of pharmacological and non-pharmacological therapies for migraine prophylaxis using a new frequentist method.

- The results of this systematic review will help clinicians and migraine patients to choose suitable treatment options.

- Owing to the difficulty of locating all the effective interventions for migraine prophylaxis, which may be one of the major limitations of this metaanalysis, we will comprehensively review the guidelines for the recommended interventions.

quality of life, thus leading to lower incomes and society status, as well as worse emotional status. $^{8}$

Prophylactic treatments are necessary if the migraine attacks affect the quality of life of a patient. ${ }^{9}$ Pharmacological treatments are the primary choice by many migraine patients, since they are convenient and effective. Systematic reviews and guidelines recommended several pharmacological treatments for migraine prophylaxis, such as antiepileptic drugs (AEDs) ${ }^{10-12}$ The use of topiramate, a kind of AED, at a daily dose of $50 \mathrm{mg}$ demonstrated a favourable effect in reducing the migraine days. ${ }^{13}$ However, many patients reported intolerance of the prophylactic drugs because of side effects. ${ }^{12}$ Some patients reported a tolerance to the beneficial effects of prophylactic drugs for migraine treatment, also after a continuous drug treatment. ${ }^{14}$ So these patients turned to seek nonpharmacological treatments. Recently, nonpharmacological treatment for migraine prophylaxis are promisingly developed, like acupuncture, cognitive therapy, etc. Several randomised controlled trials (RCTs) and systematic reviews confirmed the effectiveness of some non-pharmacological treatments. ${ }^{15-18}$ If we take acupuncture as an example, a total of 12 sessions of acupuncture will reduce at least 
half of the migraine attacks in $51 \%$ of the patients with moderate or severe migraine headaches. ${ }^{19}$ A clinical question was raised: which prophylactic treatment is the best choice for migraine patients-pharmaceuticals or non-pharmacological treatments? Another question would be whether different types of pharmaceuticals vary in their effectiveness, and the same question would also be considered for the non-pharmacological treatments. However, these questions were not fully answered, although recently two network meta-analyses were conducted to answer one of the above questions. ${ }^{10}{ }^{18}$ Providing direct and indirect evidence (the comparative effectiveness) is meaningful for helping the patients and clinicians to make their choice of the best prophylactic treatment. Therefore, we will conduct an umbrella systematic review and network meta-analysis to answer the following questions: (1) Which is the best treatment for reducing the number of migraine attacks in all the treatments? (2) Which treatment would yield the lowest proportion of patients with adverse events?

\section{METHODS}

\section{Study design}

Considering the large number of pharmacological and non-pharmacological therapies for migraine prophylaxis and the availability of numerous systematic reviews that examined the efficacy of these interventions, we will conduct an umbrella systematic review to identify eligible RCTs. ${ }^{20}$ In brief, we will perform a search for all interventional systematic reviews that examined the effectiveness of any treatments for migraine prophylaxis. Then we will exclude the interventions that are not recommended for migraine prophylaxis in the guidelines, to ensure that the interventions included in our network meta-analysis will all be positive comparators, since a superiority over a non-effective intervention is meaningless and misleading. We will screen RCTs from the available systematic reviews and extract information from the RCTs to run a network meta-analysis. This review has been registered at PROSPERO (ID: CRD42015015297). The procedure of this review is shown in figure 1 .

\section{Search strategy}

We will search the electronic databases MEDLINE, EMBASE and the Cochrane library for systematic reviews from inception to 2014, which examine the effectiveness of the pharmacological and non-pharmacological interventions for migraine prophylaxis. If multiple systematic reviews on the same topic (examining the same intervention for migraine prophylaxis) are found, we will use the latest version of the reviews. The interventions that we intend to include should be recommended in the guidelines that are developed by the American Academy of Neurology, the National Institute for Health and Care Excellence (NICE), the Scottish Intercollegiate Guidelines Network (SIGN) and the Canadian Headache Society. ${ }^{91-25}$
Search electronically for systematic

reviews of migraine prophylaxis

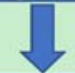

Extract RCTs from the systematic reviews

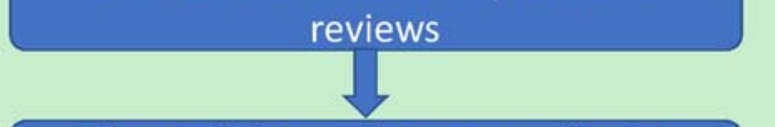

Choose interventions according to guidelines

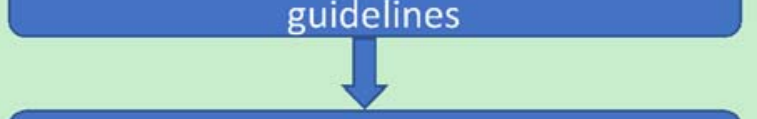

Extract data from the RCTs

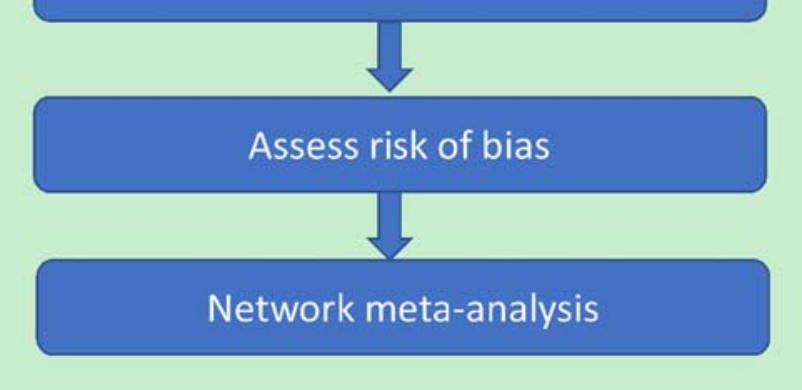

Figure 1 Flow chart of the systematic review (RCTs, randomised controlled trials).

The level of recommendation for the interventions should be moderate or high. The databases will be searched from 2005 to date. To ensure that the most recent trials will be included, we will also search RCTs testing interventions for migraine prophylaxis in the databases in the year 2014. An experienced librarian will help to develop a search strategy to find out systematic reviews that examined the effectiveness of the recommended interventions. A combination of terms of Medical Subject Headings (MeSH) and keywords will be used in the search strategy. MeSH and keywords contain 'migraine', 'systematic review' and synonymous words. A detailed search strategy is provided in table 1. Language restrictions will not be used in this review.

\section{Identification of the studies}

After a search for the systematic reviews, two independent reviewers ( $\mathrm{HZ}$ and $\mathrm{MC}$ ) will independently review the titles and abstracts for possible candidates, which will be searched and downloaded for full-text copies for further evaluation. They will also examine the full-text copies and extract the included RCTs in the reviews into a data set. Duplicate RCTs will be excluded in the data set. We will screen and include RCTs that examine the efficacy of the interventions that are listed in table 2 . These interventions are selected according to the aforementioned guidelines. For pharmacological treatments, we will include the trials using a dose recommended by the guidelines or a higher dose, to ensure that we 
Table 1 Search strategy for MEDLINE and EMBASE via the OVID database

\begin{tabular}{ll}
\hline 1 & exp migraine \\
2 & limit 1 to meta analysis \\
3 & systematic review.mp. \\
4 & 1 and 3 \\
5 & 2 or 4 \\
6 & Meta analysis \\
7 & systematic review.mp. \\
8 & 1 and $(6$ or 7$)$ \\
9 & 4 or 8 \\
10 & remove duplicates from 9 \\
11 & limit 10 to (editorial or letter) \\
12 & 10 not 11 \\
13 & limit 12 to $y r=" 2005-2014 "$ \\
\hline
\end{tabular}

include the positive comparators. If several doses of a pharmacological intervention are studied in one trial, we will include the arm with a recommended dose or a higher one. For non-pharmacological treatments, we will

Table 2 Recommended interventions by the guidelines for migraine prophylaxis*

\begin{tabular}{|c|c|}
\hline Interventions & $\begin{array}{l}\text { Minimum effective dose or } \\
\text { treatment period, mg/day }\end{array}$ \\
\hline \multicolumn{2}{|c|}{ Pharmacological interventions } \\
\hline \multicolumn{2}{|c|}{ Antiepileptics } \\
\hline Gabapentin & 900 \\
\hline Topiramate & 100 \\
\hline \multicolumn{2}{|l|}{ Antidepressants } \\
\hline Amitriptyline & 10 \\
\hline Nortriptyline & 10 \\
\hline \multicolumn{2}{|c|}{ Antihypertensives and other calcium channel blockers } \\
\hline Propranolol & 80 \\
\hline Metoprolol & 100 \\
\hline Nadolol & 80 \\
\hline Flunarizine & 10 \\
\hline Candesartan & 16 \\
\hline \multicolumn{2}{|c|}{ Vitamins/minerals/herbals } \\
\hline Riboflavin & 400 \\
\hline Coenzyme Q10 & 300 \\
\hline Magnesium & 600 \\
\hline $\begin{array}{l}\text { Butterbur (Petasites } \\
\text { hybridus root extract) }\end{array}$ & 150 \\
\hline \multicolumn{2}{|l|}{ Serotonin Antagonists } \\
\hline \multicolumn{2}{|c|}{ Non-pharmacological interventions } \\
\hline Acupuncture & - \\
\hline Relaxation training & - \\
\hline Biofeedback & - \\
\hline $\begin{array}{l}\text { Cognitive-behavioural } \\
\text { therapy }\end{array}$ & - \\
\hline \multicolumn{2}{|c|}{$\begin{array}{l}\text { *The recommended dose or treatment period was defined } \\
\text { according to the guidelines developed by the American academy } \\
\text { of neurology, the National Institute for Health and Care } \\
\text { Excellence, the Scottish Intercollegiate Guidelines Network and } \\
\text { the Canadian Headache Society. There is no standard reference } \\
\text { for defining the minimum effective treatment period of the } \\
\text { non-pharmacological interventions. }\end{array}$} \\
\hline
\end{tabular}

include trials that used the above pharmacological treatments as positive controls, and trials that used placebo pills or sham interventions as placebo controls. The RCTs that compare different types of nonpharmacological treatments without a positive comparator or placebo or sham intervention arm as control will be excluded. Those that included episodic or chronic migraine patients will be included in our systematic review. No language restrictions will be applied. The outcomes of the RCTs should include the assessment of the migraine attack frequency. If there are disagreements between the two reviewers, all the authors will discuss and solve the problems.

\section{Data extraction}

After identification of the target RCTs, two independent reviewers (JL and QC) will extract the necessary information from the included RCTs using a standard form. The form will be developed by a consensus of all the reviewers, which covers the following domains. First, we will extract the general information of the included RCTs for country, number of centres, publication source, number of participants, design (parallel/crossover), number of arms, allocation ratio, main results and conclusions. Second, we will extract characteristics of the migraine patients in the RCTs, including age, sex, duration of experience of migraine attack, and a subclassification of migraine types; if possible, we will also extract information on body mass index, use of alcohol and preventive medication during the past 3 months. Third, we will extract details of the interventions in the RCTs, which include the interventions in the experimental and control groups. For the pharmacological interventions, we will record the drug category (eg, AEDs), chemical name of the drug, dose, way of administration (eg, oral, muscular injection), the whole treatment period and the compliance of the interventions. For the nonpharmacological interventions, we will record the name of the intervention (eg, acupuncture), years of experience of the practitioners, the total number of all treatment sessions, the lasting time for each session, accompanied treatments and the overall treatment period. Fourth, we will extract the information of the outcomes. We will record the primary and secondary outcomes, measurement time points, use of headache diaries and the proportion of adverse events.

\section{Outcome assessment}

The primary aim of this study is to evaluate the recommended treatments in the guidelines for reducing the mean headache frequency in a month. Therefore, we will consider the number of days of migraine attacks or the number of migraine attacks in a month (or in a 4-week period) as the primary outcome. The primary outcome will be defined according to the International Headache Society (IHS) guideline. According to a previous review of migraine prophylaxis, the primary outcome assessed at 3-4 months after randomisation is 
considered as the most appropriate time point for effectiveness assessment. So we will include data of the primary outcome collected during 3-4 months after randomisation. If the included trials assess the primary outcome outside the time range, we will select the time point near 3-4 months after randomisation. The primary outcome assessed at the other time points will be analysed in the secondary analysis as the supporting evidence. The secondary outcome will be the proportion of the responders. The responder will be defined according to the IHS guideline, which refers to a participant with a $\geq 50 \%$ improvement in migraine attacks or a $\geq 50 \%$ reduction in number of migraine days, compared with baseline values. If the included RCTs assessed the proportion of responders at different time points, we will also select the time point at or near 3-4 months after randomisation. After the two independent reviewers (MC and $\mathrm{QC}$ ) extract the information from the included RCTs, a third reviewer (JF) will check the completeness and correctness of the extracted data of the outcome assessment, to ensure an accurate result of this study.

\section{Risk of bias assessment}

We will choose the Cochrane Collaboration's risk of bias tool to evaluate the methodological quality of RCTs. The risk of bias tool consists of six domains: sequence generation, allocation concealment, blinding, incomplete data, selective reporting and other bias. Two independent reviewers (MC and QC) will independently evaluate the quality of RCTs. Sequence generation will be considered as adequate if central randomisation or tables of random numbers are used. Allocation concealment will be considered as adequate if central randomisation or sealed envelopes are used. We will consider blinding as adequate if participants, outcome assessors and statisticians are blinded from the group assignment. The other domains will be assessed exactly as the criteria of the risk of bias tool. A summary of risk of bias of all the six domains will be provided for each trial. We choose to consider sequence generation, allocation concealment and blinding as the key essential domains to score the overall quality of a trial. Discrepancies among the two reviewers ( $\mathrm{MC}$ and $\mathrm{QC}$ ) will be solved by discussion, or will be judged by a third reviewer $(\mathrm{DH})$.

\section{Statistical analysis}

The data for statistical analysis will be extracted into an Excel file. The primary outcome is continuous data, so we will calculate the effect size of the interventions using the standardised mean difference (SMD). For trials that present mean values of each time point, we will use the primary outcome adjusted by the baseline values. If the trials present the value of the primary outcome changing from baseline, we will calculate the SMD directly. We will calculate the $95 \%$ CI for each single SMD, and the results will be pooled using the random-effect model. The proportion of responders represents dichotomous data, so we will calculate the effect size using the relative ratio (RR). The RR and the $95 \% \mathrm{CI}$ of each intervention will be calculated and pooled using the random-effect model.

The network meta-analysis will be conducted using the 'netmeta' package in the $\mathrm{R}$ software (http://www. r-project.org/), to combine direct and indirect evidence of interventions for migraine prophylaxis. ${ }^{26}$ The package is developed on the basis of the frequentist method, using the graph-theoretical method developed according to the electrical network theory. ${ }^{27}$ The first advantage of this method is that it can combine direct and indirect evidence in trials with more than two study arms. Multiarm studies are often included in a network meta-analysis. In these studies, the treatment effects on different comparisons are correlated, which is not fully addressed by the generalised linear mixed models ${ }^{28}$ or the Bayesian Markov Chain Monte Carlo method ${ }^{29} 30$ that is commonly used for network meta-analysis. The 'netmeta' package accounts for the correlated treatment effects by reweighting all comparisons of each multiarm study. The second advantage of this method is that it provides solutions for testing the consistency of the network using Cochrane's $Q$ statistics and finding out the reasons for the consistency by a net-heat plot. So we will use this method to address the consistency of the network. If the data are not suitable to carry out the synthesis, we will perform a descriptive review and summarise the evidence. The evidence strength will be assessed using the GRADE method generated by the Cochrane library. A funnel plot will be drawn to detect if there is any publication bias.

\section{Dealing with missing data}

There will be missing data in the trials that we included. We will first contact the authors to ask for original data by email or phone calls, if possible. If the original data are not available, we will try to calculate the data through the available coefficients; for example, we will calculate the SD from the 95\% CI, $\mathrm{p}$ or $\mathrm{t}$ values. Imputations of the data will be tested in the following sensitivity analysis.

\section{Subgroup analysis}

To address the potential heterogeneity and inconsistency across trials, we will perform a subgroup analysis. This include subtypes of migraine (migraine with aura, migraine without aura, menstrual-related migraine, chronic migraine), blinding method (open trial, single blind for participants, double blind for both participants and care providers), quality of evidence (high risk, unclear of the risk and low risk), duration of migraine and mean age of the participants. Assessment of migraine outcome is subjective, so we will consider the blinding method as the most important subgroup analysis. Meta-regression models will be used to quantify the difference between subgroups and test for statistical significance. 


\section{Sensitivity analysis}

Sensitivity analysis will be performed to first address whether the combined estimates of the interventions are dominated by one or several trials, especially those with a high risk of bias. Then we will exclude the trials to test the robustness of our study result. Second, we will test whether the imputation of the missing values affects the result of the meta-analysis. We will also test different coefficients that are used to impute the missing value; if both $\mathrm{SE}$ and $95 \% \mathrm{CI}$ are available to calculate $\mathrm{SD}$, we will test which is better.

\section{DISCUSSION}

This network meta-analysis is expected to provide a ranking of the interventions from guideline recommendations for migraine prophylaxis, based on comparative effectiveness evidence. The migraine patients and their physicians could choose their best preferences, after considering the combined effect estimates, known side effects and the costs. We also hope that the result would be of interest to the policymakers of health insurance; this might help them to make a better choice of the interventions that should be covered by insurance.

Acknowledgements The authors would like to thank the librarian Yulan Ren for helping us to develop the search strategy.

Contributors $\mathrm{HZ}, \mathrm{MC}, \mathrm{QC}, \mathrm{DH}$ and JF contributed to the conception and design of the study protocol. The search strategy was developed and run by $\mathrm{HZ}$ and $\mathrm{MC}$, who will also screen the title and abstract of the studies after running the search strategy. $\mathrm{HZ}$ and $\mathrm{MC}$ will also screen full copies of the remaining studies after title and abstract selection, while $\mathrm{JL}$ and $\mathrm{QC}$ will extract information of the identified studies; QC will check the data entry for accuracy and completeness. DH and JF will give advice for data analysis and presentation. All the authors drafted and revised this study protocol and approved it for publication.

Funding This study is supported by the Open Research Fund of Zhejiang First-foremost Key Subject-Acupuncture \& Tuina (grant number (ZTK2010A16) and National Natural Science Foundation (grant numbers (81102656 and 81473777).

Competing interests None declared.

Provenance and peer review Not commissioned; peer reviewed for ethical and funding approval prior to submission.

Open Access This is an Open Access article distributed in accordance with the Creative Commons Attribution Non Commercial (CC BY-NC 4.0) license, which permits others to distribute, remix, adapt, build upon this work noncommercially, and license their derivative works on different terms, provided the original work is properly cited and the use is non-commercial. See: http:// creativecommons.org/licenses/by-nc/4.0/

\section{REFERENCES}

1. Lipton RB, Stewart WF, Diamond S, et al. Prevalence and burden of migraine in the United States: data from the American Migraine Study II. Headache 2001;41:646-57.

2. Lipton R, Bigal M, Diamond Mo, et al. Migraine prevalence, disease burden, and the need for preventive therapy. Neurology 2007:68:343-9.

3. Steiner T, Scher A, Stewart W, et al. The prevalence and disability burden of adult migraine in England and their relationships to age, gender and ethnicity. Cephalalgia 2003;23:519-27.
4. Wang SJ, Fuh JL, Young YH, et al. Prevalence of migraine in Taipei, Taiwan: a population-based survey. Cephalalgia 2000;20:566-72.

5. Cheung RT. Prevalence of migraine, tension-type headache, and other headaches in Hong Kong. Headache 2000;40:473-9.

6. Yu S, Liu R, Zhao G, et al. The prevalence and burden of primary headaches in China: a population-based door-to-door survey. Headache 2012;52:582-91.

7. Landy SH, Runken MC, Bell CF, et al. Assessing the impact of migraine onset on work productivity. J Occup Environ Med 2011;53:74-81.

8. Lantéri-Minet M, Duru G, Mudge M, et al. Quality of life impairment, disability and economic burden associated with chronic daily headache, focusing on chronic migraine with or without medication overuse: a systematic review. Cephalalgia 2011;31:837-50.

9. Silberstein S, Holland S, Freitag F, et al. Evidence-based guideline update: pharmacologic treatment for episodic migraine prevention in adults Report of the Quality Standards Subcommittee of the American Academy of Neurology and the American Headache Society. Neurology 2012;78:1337-45.

10. Shamliyan TA, Choi J-Y, Ramakrishnan R, et al. Preventive pharmacologic treatments for episodic migraine in adults. $J$ Gen Intern Med 2013;28:1225-37.

11. Pringsheim T, Davenport WJ, Mackie G, et al. Systematic review: medications for migraine prophylaxis-section II. Can J Neurol Sci 2012;39:2-S8.

12. Fenstermacher $\mathrm{N}$, Levin $\mathrm{M}$, Ward $\mathrm{T}$. Pharmacological prevention of migraine. BMJ 2011;342:540-3.

13. Mulleners WM, McCrory DC, Linde M. Antiepileptics in migraine prophylaxis: an updated Cochrane review. Cephalalgia 2015; 35:51-62.

14. Rizzoli $P$, Loder EW. Tolerance to the beneficial effects of prophylactic migraine drugs: a systematic review of causes and mechanisms. Headache 2011;51:1323-35.

15. Varkey E, Cider Å, Carlsson J, et al. Exercise as migraine prophylaxis: a randomized study using relaxation and topiramate as controls. Cephalalgia 2011;31:1428-38.

16. $\mathrm{Li} \mathrm{Y}$, Zheng $\mathrm{H}$, Witt $\mathrm{CM}$, et al. Acupuncture for migraine prophylaxis: a randomized controlled trial. CMAJ 2012;184:401-10.

17. Kasle D, Kligler B. Systematic review of acupuncture versus medication for migraine prophylaxis. J Altern Complement Med 2014;20:A42-3.

18. Meissner K, Fässler M, Rücker G, et al. Differential effectiveness of placebo treatments: a systematic review of migraine prophylaxis. JAMA Intern Med 2013;173:1941-51.

19. Linde K, Streng $A$, Jürgens $S$, et al. Acupuncture for patients with migraine: a randomized controlled trial. JAMA 2005;293:2118-25.

20. Higgins J, Green S. Cochrane handbook for systematic reviews of interventions. Version 5.1. 0. The Cochrane Collaboration, 2011, 2013.

21. Holland S, Silberstein SD, Freitag F, et al. Evidence-based guideline update: NSAIDs and other complementary treatments for episodic migraine prevention in adults: report of the Quality Standards Subcommittee of the American Academy of Neurology and the American Headache Society. Neurology 2012;78:1346-53.

22. Kennis K, Kernick D, O'Flynn N. Diagnosis and management of headaches in young people and adults: NICE guideline. $\mathrm{Br} J$ Gen Pract 2013;63:443-5.

23. Carville S, Padhi S, Reason T, et al. Diagnosis and management of headaches in young people and adults: summary of NICE guidance. BMJ 2012;345:e5765.

24. Pringsheim T, Davenport W, Mackie G, et al. Canadian Headache Society guideline for migraine prophylaxis. Can J Neurol Sci 2012;39(2 Suppl 2):S1-59.

25. Duncan CW, Watson DP, Stein A. Diagnosis and management of headache in adults: summary of SIGN guideline. BMJ 2008;337: a2329.

26. Rücker G, Schwarzer G, Krahn U, et al. netmeta: network meta-analysis with $R$. R package version 0.5-0. http://CRAN R-project.org/package=netmeta.

27. Rücker G. Network meta-analysis, electrical networks and graph theory. Res Synth Methods 2012;3:312-24.

28. Lumley T. Network meta-analysis for indirect treatment comparisons. Stat Med 2002;21:2313-24.

29. Gilks WR. Markov chain Monte Carlo. Wiley Online Library, 2005.

30. Smith TC, Spiegelhalter DJ, Thomas A. Bayesian approaches to random-effects meta-analysis: a comparative study. Stat Med 1995;14:2685-99. 